\title{
NONHOLONOMIC MOTION PLANNING USING TRIGONOMETRIC SWITCH INPUTS
}

\author{
$\mathrm{Li}, \mathrm{L}$. \\ School of Electromechanical Engineering, Baoji University of Arts and Sciences, Baoji, China \\ E-Mail: leeliang@126.com
}

\begin{abstract}
In this paper we present a local motion planning law called trigonometric switch inputs which can steer the chained form system to the final positions, at least locally, around the initial positions. This method steers the system step by step instead of steering all states in one step. The advantages of trigonometric switch inputs law are that the motion trajectories are quite smooth and have less oscillation and lower computational costs, all of which is beneficial for the application of the time scale transformation technique and improvement of motion efficiency of the system. A two-wheeled mobile robot system is steered by this new motion planning law to illustrate the practical application. Finally, simulations with the time scale transformation technique and experiments with the mobile robot verify the feasibility and effectiveness of trigonometric switch inputs law.

(Received, processed and accepted by the Chinese Representative Office.)
\end{abstract}

Key Words: Chained Form Conversion, Motion Planning, Trigonometric Switch Input, Nonholonomic System, Time Scale Transformation

\section{INTRODUCTION}

Nonholonomic motion planning designs an appropriate bounded input planning path to steer the system from an initial position to a desired position over a finite period of time. In recent years, there have been many works involving nonholonomic motion planning. Motion planning of a chained form system in particular has become an important research field because chained form conversion provides powerful tools to steer a class of nonholonomic systems. Furthermore, motion planning for some nonholonomic systems requires high computational costs in dealing with complicated kinematic equations that cannot be converted into chained form, as in reference [1]. In reference [2], a set of sufficient conditions for conversion into chained form were given. Reference [3] addresses the chained form conversion of a car with passive trailers. Two different controllable joint manipulators were designed and controlled based on chained form conversion in references [4-6].

The chained form system is easily integrated and can be steered by existing motion planning laws. Actually, many practical nonholonomic systems, including wheeled mobile robots, can be converted into a chained form system through coordinate transformation and input feedback transformation. Many useful motion planning laws have been proposed. Murray and Sastry introduced a sinusoidal input control law based on optimal control theory for a chained form system [7]. Each state can move to the final value step by step utilizing the periodicity of sinusoidal function. Tilbury and Murray make a detailed discussion about overparameter sinusoidal, piecewise constant and time polynomial inputs law for motion planning of a chained form system in [8], Sekhavat and Laumond in [9], respectively. Nakamura et al. proposed an optimal three-point trailer system and applied time polynomial inputs and sinusoidal input laws to steer the new mechanism [10]. Measurement and control system for underactuated manipulator is described in [11]. The polynomial input control for an under actuated manipulator was studied in [12]. Chelouah et al. demonstrated the effectiveness of the digital control method through two classic examples: the car with one trailer and the hopping robot [13]. Li et al. presented a switch control method by use of bang-bang inputs to 
calculate the sequences of switching time [14]. A curve fitting approach for nonholonomic motion planning was proposed in [15]. However, use of sinusoidal inputs and polynomial inputs has high oscillation. Piecewise constant inputs and bang-bang inputs can steer the system to desired positions, but the trajectory is discontinuous. Although over-parameter sinusoidal inputs can obtain a smooth trajectory with low oscillation by searching for an appropriate value in parametric space, integral expressions of the states are too complicated when the system is up to dimension 5.

Building on the fore mentioned studies, we propose a trigonometric switch inputs law for motion planning of chained form. This method combines the trigonometric functions with discrete control law which can effectively solve the problems of discontinuity at switching time for piecewise constant inputs and bang-bang inputs and high oscillation for sinusoidal and over-parameter sinusoidal inputs. In comparison with over-parameter sinusoidal inputs, trigonometric switch inputs have a simple integral expression with low computational costs. We apply the trigonometric switch inputs law to a two-wheeled mobile robot whose kinematic equations can be converted into chained form. Simulation and experiment results prove the validity of the presented motion planning law.

This paper is organized as follows. Section 2 gives a detailed introduction to the trigonometric switch inputs law. Section 3 illustrates the application for motion planning of a mobile robot. The experiment results are shown in Section 4, and conclusions and future research projects are given in Section 5.

\section{TRIGONOMETRIC SWITCH INPUTS}

The chained form state equation of $n$ dimension with two inputs is expressed as follows:

$$
\left\{\begin{array}{l}
\dot{z}_{1}=v_{1} \\
\dot{z}_{2}=v_{2} \\
\dot{z}_{3}=z_{2} v_{1} \\
\cdots \\
\dot{z}_{n}=z_{n-1} v_{1}
\end{array}\right.
$$

In order to simplify control, the motion of the chained form should be disassembled into basis motions because of the chained relation between the variation of one coordinate and the value of the previous coordinate. In this section, we present the trigonometric switch inputs law to steer the chained form system and an application of this motion planning law for a twowheeled mobile robot.

The problem we address is that given a chained form system with an initial state $Z^{0}=\left[z_{1}^{0}, z_{2}^{0}, \ldots, z_{n}^{0}\right]^{T}$ and a desired state $Z^{f}=\left[z_{1}^{f}, z_{2}^{f}, \ldots, z_{n}^{f}\right]^{T}$, the trigonometric switch inputs $\left[0, v_{2}\right]^{T}=[0,1-\cos \omega t]^{T}$ and $\left[v_{1}, 0\right]^{T}=[1-\cos \omega t, 0]^{T}$ will steer the chained form system from an initial state $Z^{0}$ to the desired state $Z^{f}$ after finite time $T$.

Theorem 1: $n$ dimensional chained form system can be steered locally around an initial state $Z^{0}$ by trigonometric switch inputs with $2(n-2)+1$ times of switch.

Proof: The total time $T$ is equally divided into $2(n-2)+1$ successive time intervals, then the length of each time interval is $\eta=T /(2(n-2)+1)$.

In odd numbered time intervals between time $t_{2 i}$ and $t_{2 i+1}$, each time interval can also be expressed as $\eta=t_{2 i+1}-t_{2 i}, i \in\{0,1,2, \ldots, n-2\}$. Then the trigonometric switch inputs applied to chained form system (1) over the odd numbered time interval $\left[t_{2 i}, t_{2 i+1}\right]$ are determined by:

$$
\left\{\begin{array}{l}
v_{1}=0 \\
v_{2}=c_{2 i+1}(1-\cos \omega t)
\end{array} \quad t \in\left[t_{2 i}, t_{2 i+1}\right]\right.
$$


where $C_{2 i+1}$ are undetermined coefficients, $\omega$ is the angular frequency which is equal to $\omega=2 \pi / \eta$. Obviously, control inputs (2) are step-by-step over odd numbered time intervals. By direct integration, the chained form system (1) at time $t_{2 i+1}$ is evaluated to yield:

$$
\left\{\begin{array}{l}
z_{1}\left(t_{2 i+1}\right)=z_{1}\left(t_{2 i}\right) \\
z_{2}\left(t_{2 i+1}\right)=c_{2 i+1} \eta+z_{2}\left(t_{2 i}\right) \\
z_{3}\left(t_{2 i+1}\right)=z_{3}\left(t_{2 i}\right) \\
\cdots \\
z_{n}\left(t_{2 i+1}\right)=z_{n}\left(t_{2 i}\right)
\end{array}\right.
$$

Similarly, in even numbered time intervals between time $t_{2 j+1}$ and $t_{2 j+2}$, and time interval is determined by $\eta=t_{2 j+2}-t_{2 j+1}, j \in\{0,1,2, \ldots, n-3\}$. Therefore, the trigonometric switch inputs over the even numbered time interval $\left[t_{2 j+1}, t_{2 j+2}\right]$ can be expressed as follows:

$$
\left\{\begin{array}{l}
v_{1}=c_{2 j+2}(1-\cos \omega t) \\
v_{2}=0
\end{array} \quad t \in\left[t_{2 j+1}, t_{2 j+2}\right]\right.
$$

where $c_{2 j+2}$ are undetermined coefficients. The control inputs can be applied to the chained form state Eqs. (1) over the even numbered time intervals. The endpoint of the chained form at time $t_{2 j+2}$ can be integrated as follows:

$$
\left\{\begin{array}{l}
z_{1}\left(t_{2 j+2}\right)=c_{2 i+2} \eta+z_{1}\left(t_{2 j+1}\right) \\
z_{2}\left(t_{2 j+2}\right)=z_{2}\left(t_{2 j+1}\right) \\
z_{3}\left(t_{2 j+2}\right)=c_{2 j+2} z_{2}\left(t_{2 j+1}\right) \eta+z_{3}\left(t_{2 j+1}\right) \\
\cdots \\
z_{n}\left(t_{2 j+2}\right)=\sum_{k=1}^{n-2} \frac{\left(c_{2 j+2} \eta\right)^{k} z_{n-k}\left(t_{2 j+1}\right)}{k !}+z_{n}\left(t_{2 j+1}\right)
\end{array}\right.
$$

The desired state $Z^{f}$ of the chained form system is the configuration reached from the initial state $Z^{0}$ by applying the trigonometric switch inputs. Then, the desired state $Z^{f}$ at time $T$ can be calculated by iterative operation via (3) and (5).

$$
\left\{\begin{array}{l}
z_{1}^{f}=\sum_{j=0}^{n-3} c_{2 j+2} \eta+z_{1}^{0} \\
z_{2}^{f}=\sum_{i=0}^{n-2} c_{2 i+1} \eta+z_{2}^{0} \\
z_{3}^{f}=\sum_{i=0}^{n-3}\left(\sum_{j=i}^{n-3} c_{2 j+2} \eta\right) c_{2 i+1} \eta+\sum_{j=0}^{n-3} c_{2 j+2} \eta z_{2}^{0}+z_{3}^{0} \\
\ldots \\
z_{n}^{f}=\sum_{i=0}^{n-3} \frac{\left(\sum_{j=i}^{n-3} c_{2 j+2} \eta\right)^{n-2}}{(n-2) !} c_{2 i+1} \eta+\sum_{k=1}^{n-2} \frac{\left(\sum_{j=0}^{n-3} c_{2 j+2} \eta\right)^{k}}{k !} z_{n-k}^{0}+z_{n}^{0}
\end{array}\right.
$$

Obviously, there are $2(n-2)+1$ undetermined coefficients which can be expressed by two coefficient vectors $C_{0}=\left[c_{1}, c_{3}, \ldots, c_{2 n-3}\right]^{T}$ and $C_{E}=\left[c_{2}, c_{4}, \ldots, c_{2 n-4}\right]^{T}$ and only $n$ algebraic equations in (6) such that the solution of those coefficients is not unique. In addition, undetermined coefficients $C_{E}$ must be satisfied as:

$$
\sum_{j=0}^{n-3} c_{2 j+2}=\frac{z_{1}^{f}-z_{1}^{0}}{\eta}
$$


Consequently, a set of coefficients $C_{E}$ can be specified; then the remaining undetermined coefficients $C_{0}$ can be obtained by substituting the initial state $Z^{0}$, for the desired state $Z^{f}$ and total time $T$ into (6). From (6) and (7), Theorem 1 has been proved.

Remark 1: It should be noted that $\sum_{j=0}^{n-3} c_{2 j+2}$ are equal to zero if $z_{1}(T)=z_{1}(0)$. In other words, the coefficient vector $C_{0}$ fails to yield a solution from (6). This problem can be solved by choosing an intermediate state $Z^{c}$ which is not equal to the initial state $Z^{0}$ and the desired state $Z^{f}$. Therefore the motion planning from the initial state $Z^{0}$ to the desired state $Z^{f}$ can be dealt with by planning two paths. The first path steers the initial state $Z^{0}$ to the intermediate state $Z^{c}$. The second path joins the intermediate state $Z^{c}$ with the desired state $Z^{f}$. Furthermore, the control inputs $[1-\cos \omega t, 0]^{T}$ are used to steer the chained form system to the intermediate state in advance, then the intermediate state can be viewed as a new initial state. Finally, the coefficient vector $C_{0}$ can be solved by (6). Therefore, the trigonometric switch inputs law can steer the chained form system to the desired state $Z^{f}$ which is a configuration of the local neighbourhood of the initial state $Z^{0}$ if the control inputs switch between (2) and (4).

Remark 2: Because appropriate values can reduce the overshoot of the planning path, a set of coefficients $C_{E}$ must be carefully chosen. Of course, numerical computation can find better values by searching $C_{E}$ space. Total time $T$ can also be divided into unequal intervals, and the equal intervals chosen here is for the convenience of computation. In addition, a simulation of the time scale transformation technique will be shown in subsequent sections.

Remark 3: The trigonometric switch inputs law is used to ensure the continuity of velocity and acceleration curves of the chained form system (1). Displacement and velocity curves of states are smooth and differentiable, allowing for the smooth movement between two given configurations in the system.

\section{APPLICATION FOR TWO-WHEELED MOBILE ROBOT}

\subsection{Chained conversion of two-wheeled mobile robot}

The two-wheeled mobile robot is a typical example of a nonholonomic system [16]. The nonholonomic constraint arises due to the rolling without slipping contact between the wheels and the ground. A two-wheeled mobile robot is shown in Fig. 1.

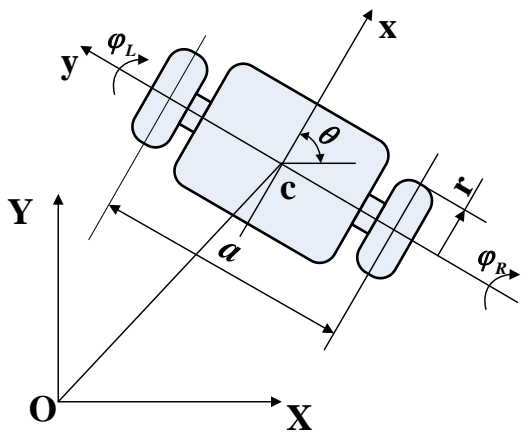

Figure 1: Two-wheeled mobile robot.

The kinematic equations of the two-wheeled mobile robot can be expressed as follows:

$$
\dot{q}=f(q) \cdot u
$$

where $q=[x, y, \theta]^{T}, f(q)=\left[\begin{array}{cc}\cos \theta & 0 \\ \sin \theta & 0 \\ 0 & 1\end{array}\right], u=\left[u_{1}, u_{2}\right]^{T} . x, y$ are the Cartesian coordinates of the 
centre point of the two-wheeled mobile robot, $\theta$ is the heading angle, and $u_{1}$ and $u_{2}$ are straight and angular velocities of the two-wheeled mobile robot at its centre point. The relationship between $u_{1}, u_{2}$ and angular velocities of the two driving wheels is:

$$
\left[\begin{array}{l}
u_{1} \\
u_{2}
\end{array}\right]=\left[\begin{array}{cc}
\frac{r}{2} & \frac{r}{2} \\
\frac{r}{a} & -\frac{r}{a}
\end{array}\right] \cdot\left[\begin{array}{c}
\dot{\varphi}_{R} \\
\dot{\varphi}_{L}
\end{array}\right]
$$

where $\dot{\varphi}_{R}$ and $\dot{\varphi}_{L}$ represent the angular velocities of the right and left wheels of the mobile robot, respectively. $a$ is the distance between the two wheels, and $r$ is the radius of the two driving wheels. The kinematic Eq. (8) of two-wheeled mobile robot can be converted into a two-input chained form system with three states by coordinate transformation and input feedback transformation as follows:

$$
\begin{gathered}
\left\{\begin{array}{l}
z_{1}=x \\
z_{2}=\tan \theta \\
z_{3}=y
\end{array}\right. \\
\left\{\begin{array}{l}
v_{1}=\cos \theta \cdot u_{1} \\
v_{2}=\frac{1}{\cos ^{2} \theta} \cdot u_{2}
\end{array}\right.
\end{gathered}
$$

Since the trigonometric switch inputs $v_{1}$ and $v_{2}$ is step-by-step, the straight and angular velocities of the two-wheeled mobile robot will be switching alternately. Therefore, motion planning of the robot from an initial position $\left[x^{0}, y^{0}, \theta^{0}\right]^{T}$ to the desired position $\left[x^{f}, y^{f}, \theta^{f}\right]^{T}$ can be divided into three steps. The first step is rotary movement around with its centre point having different velocities between the two driving wheels. The second step, which steers the two-wheeled mobile robot to the desired coordinate of centre point $\left[x^{f}, y^{f}\right]^{T}$ from an initial coordinate of centre point $\left[x^{0}, y^{0}\right]^{T}$, is linear motion with the same angular velocities between the two driving wheels. Lastly, the mobile robot rotates to achieve the desired heading angle $\theta^{f}$.

\subsection{Simulations of two-wheeled mobile robot}

By setting the initial position as $\left[x^{0}, y^{0}, \theta^{0}\right]^{T}=[0,1,0]^{T}$ and the final position as $\left[x^{f}, y^{f}, \theta^{f}\right]^{T}=$ $[5,0, \pi / 4]^{T}$, the trigonometric switch inputs law is applied to the two-wheeled mobile robot whose kinematic equations can be converted into three-dimension chained form. The structural parameters ( $a=0.2$ and $r=0.093$, unit: meter) depend on a prototype of the twowheeled mobile robot which will be shown in section 4 . Since $z_{1}(0) \neq z_{1}(\mathrm{~T})$, motion planning of the two-wheeled mobile robot requires three steps of switching velocity inputs according to theorem 1. For simplicity, $T$ is divided into three equal time intervals $\eta$ which is equal to 1 .

In the first and last time intervals, (2) is applied to the mobile robot as the control inputs. In the second time interval, (4) is used to steer the system (10). The value of $c_{2}$ is determined by (7), and the remaining undetermined coefficients $c_{1}$ and $c_{2}$ can be solved by substituting $c_{2}$ into (6). The control inputs can be addressed by solving (2) and (4). Finally, a planning trajectory and control inputs curves of the system (1) can be mapped back into state space $[x, y, \theta]^{T}$ by solving the inverse transformation of (10) and (11). The simulation results are illustrated in Figs. 2 and 3. 


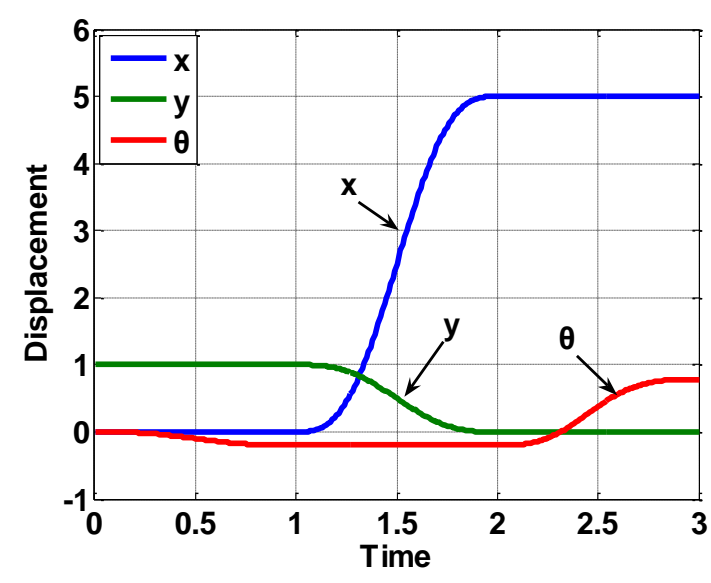

Figure 2: Displacement of mobile robot.

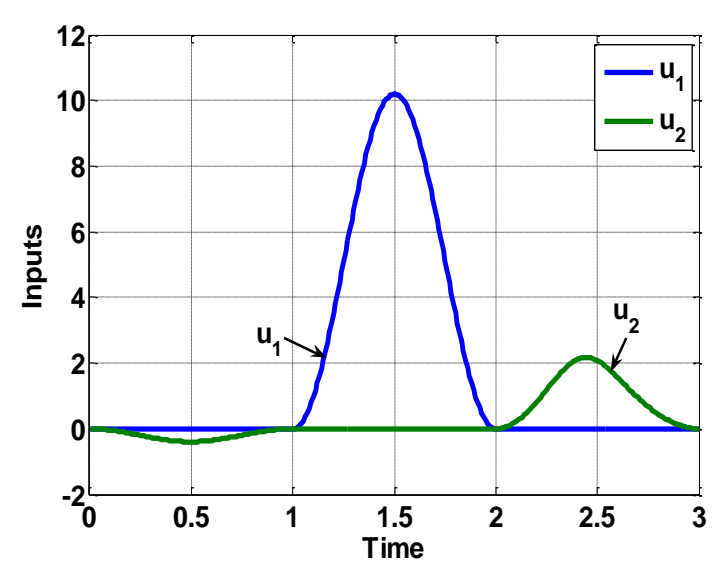

Figure 3: Inputs of mobile robot.

It is noted that the total time $T$ equals 3 seconds and the slope of the planning path and velocity curves of the second time interval is too high in Figs. 2 and 3. The time scale transformation technique can be used to deal with these problems. Assuming a new time scale as $t_{s}$ yields the following equation:

$$
t_{s}=S_{s} t
$$

where $S_{s}$ is the scaling factor which is assumed to be a constant during a time interval. Then (8) can be rewritten as:

$$
\begin{gathered}
\dot{q}_{s}=\frac{d q_{s}}{d t_{s}}=f\left(q_{s}\right) \frac{1}{S_{s}} u=f\left(q_{s}\right) u_{s} \\
\Rightarrow u_{s}=\frac{1}{S_{s}} u \\
\quad q(t)=q_{s}\left(S_{s} t\right)=q_{s}\left(t_{s}\right) \\
\Rightarrow \dot{q}=S_{s} \dot{q}_{s}
\end{gathered}
$$

where $q_{s}$ and $u_{s}$ are the scaled coordinates and inputs. Obviously, (13) indicates the original velocities $u$ are the $S_{s}$ times of the scaled velocities $u_{s}$, and (14) shows that the scaled trajectories do not vary and the slope of the planning path will change after the time scale transformation.

There are scaling factors $\left[S_{s 1}, S_{s 2}, S_{s 3}\right]^{T}$ for scaling each time interval. Although time scale transformation can make the curves of the planning path and input velocities flatten, there may be some discontinuous points on the curves since the slope will become different at the time of change of the scaling factor $S_{s}$. One of advantages of the trigonometric switch inputs law is that the scaled curves of a planning path and velocities can always remain smooth because the slope is equal to zero at the initial position and the end position for each time interval.

The three scaled time intervals are $\eta_{s}=[5,15,10]^{T}$ by setting the scaling factors as $\left[S_{s 1}, S_{s 2}, S_{s 3}\right]^{T}=[5,15,10]^{T}$. The results of simulation 1 with time scale transformation are shown in Figs. 4 and 5. Fig. 6 illustrates the trajectories of motion planning which are carried out by two different laws. The solid curve is the path planned by trigonometric switch inputs, and the dotted curve shows the planning path calculated by polynomial inputs. Although the two-wheeled mobile robot can accurately follow two planning paths from an initial position to the desired position, the path expressed by the solid curve has almost no oscillation and basically approximates a straight line. In other words, the planning motion with trigonometric switch inputs is more efficient. 


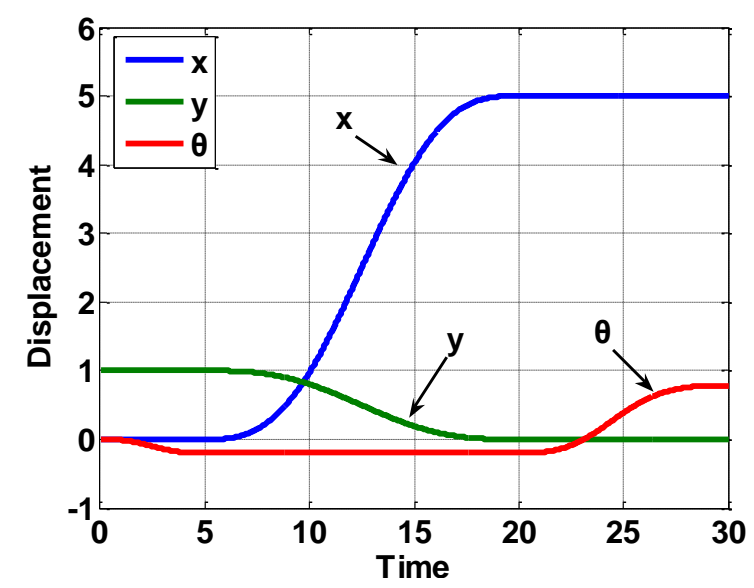

Figure 4: Displacement with time scale transformation.

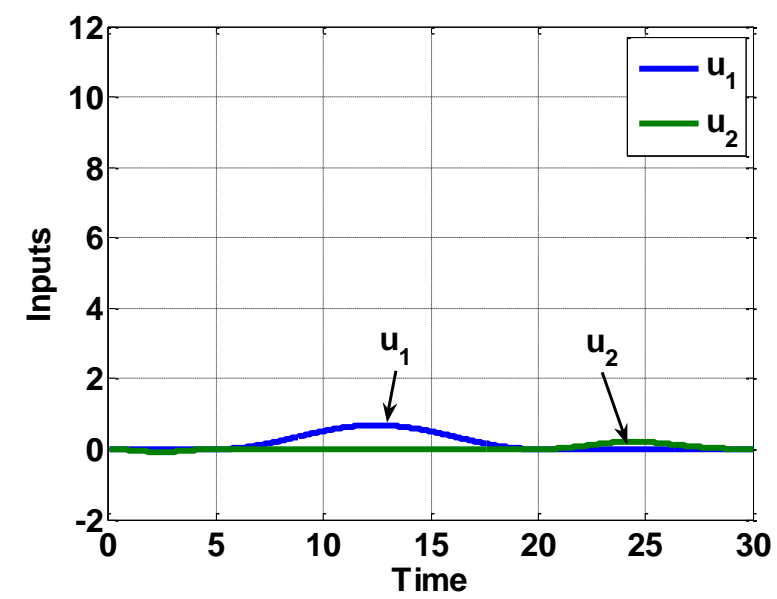

Figure 5: Input with time scale transformation.

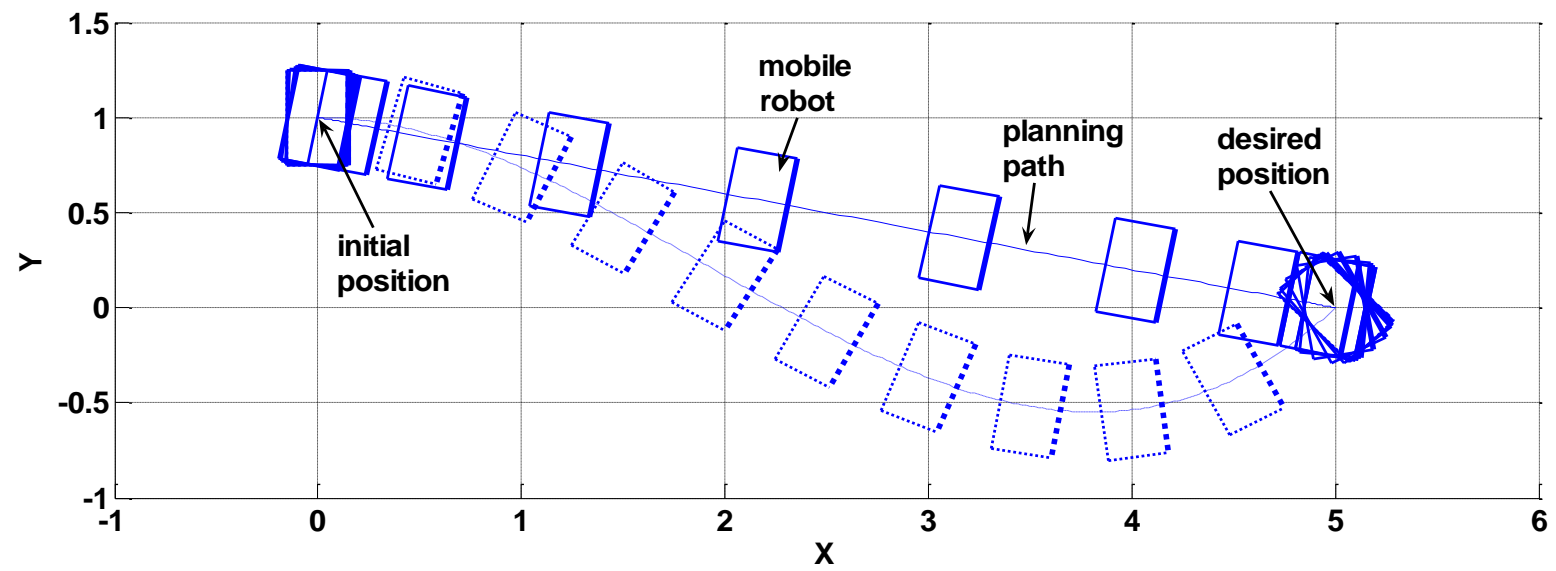

Figure 6: Trajectory of the two-wheeled mobile robot in $R^{2}$ (Simulation 1).

The second task is parallel parking of the two-wheeled mobile robot. Initial and desired positions are $[0,3, \pi / 18]^{T}$ and $[0,0,0]^{T}$, respectively. As remark 1 described in section 2 , the inputs $[(1-\cos \omega t), 0]^{T}$ can steer the two-wheeled mobile robot to an intermediate position which can be regarded as a new initial position. The four scaled time intervals are $\eta_{s}=\eta \cdot S_{s}=$ $[5,8,12,5]^{T}$. The results of simulation 2 of parallel parking are illustrated in Figs. 7 and 8 . Fig. 9 shows two different trajectories planned by the polynomial inputs law and trigonometric switch inputs law as solid and dotted lines, respectively. It can be seen that the movement of the solid curve is decomposed into straight line motion and steering motion. 
Certainly, this step-by-step motion method may seemingly cause a waste of time, but it makes the planning path simple and is more easily applied to practical equipment.

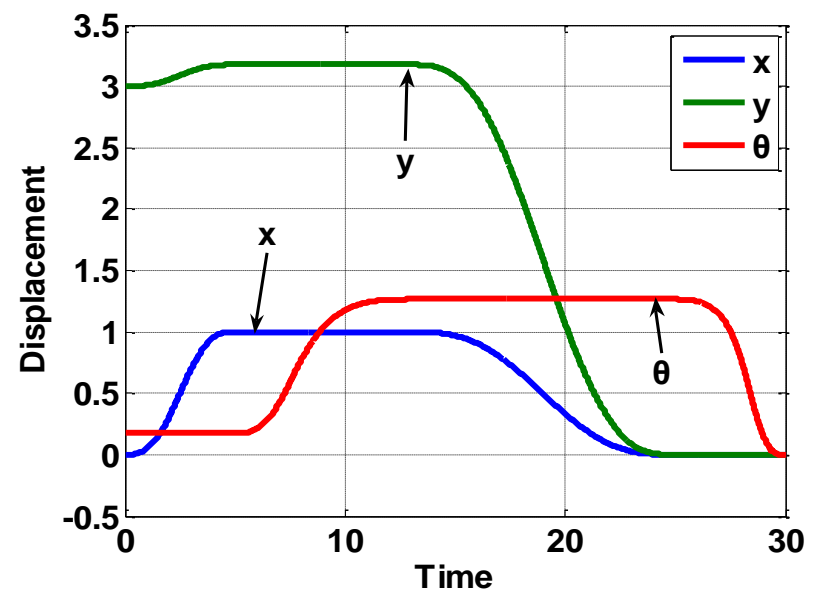

Figure 7: Displacement with time scale transformation.

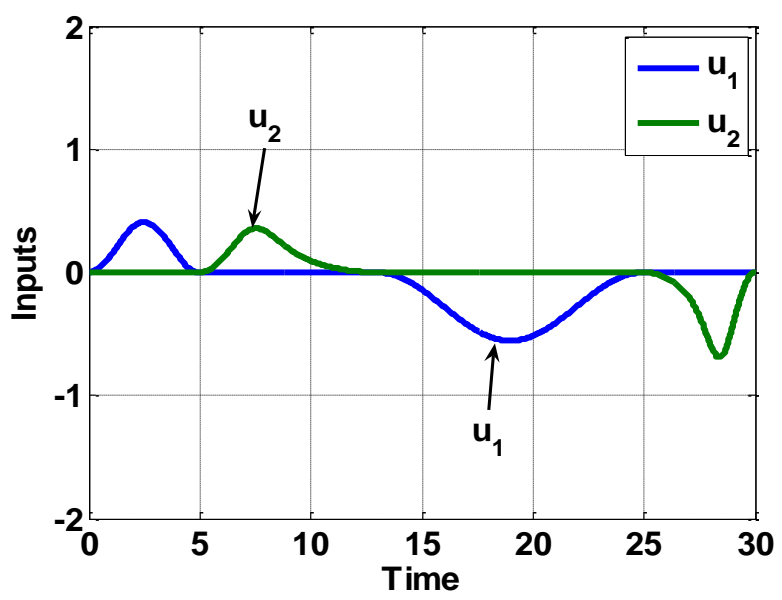

Figure 8: Inputs with time scale transformation.

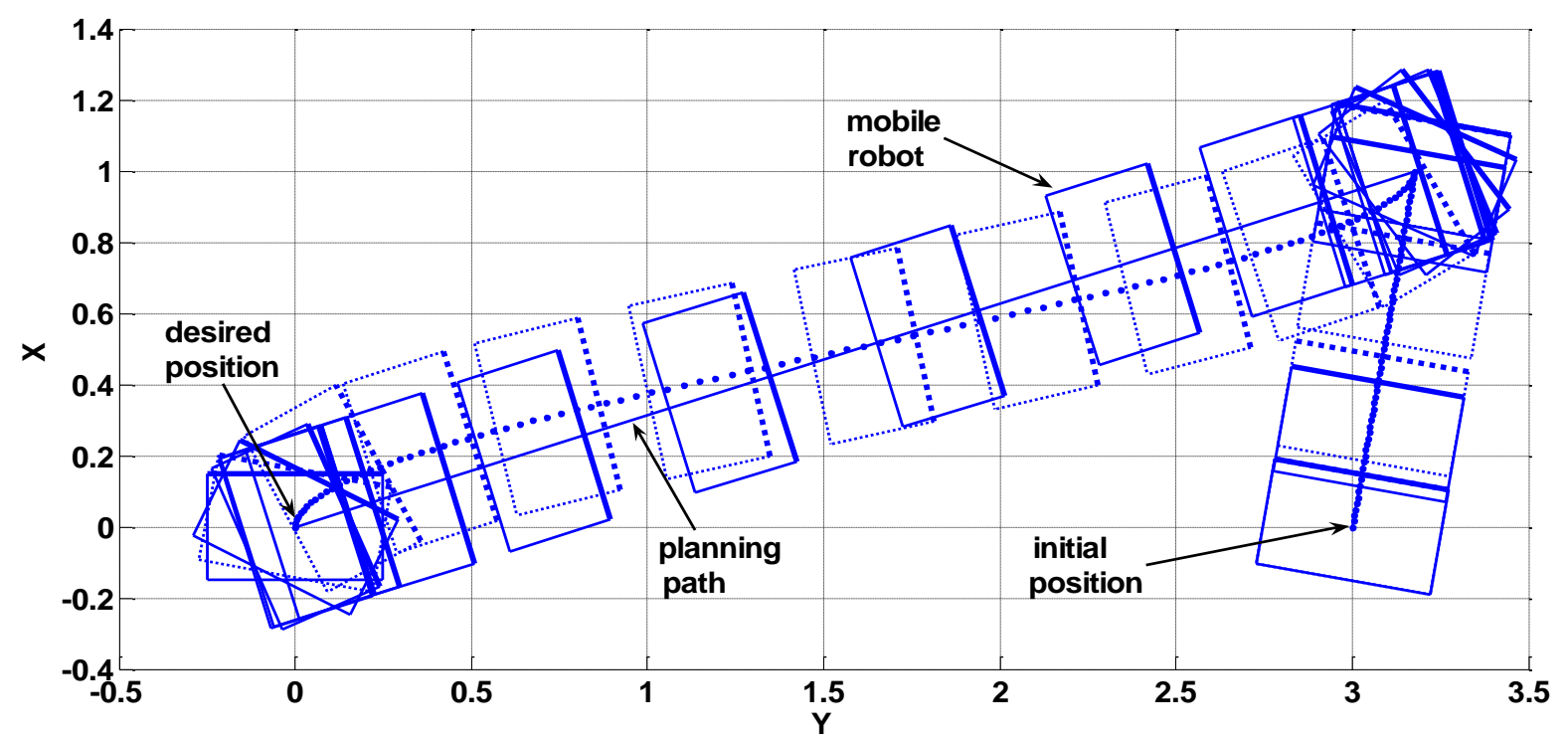

Figure 9: Trajectory of the two-wheeled mobile robot in $R^{2}$ (Simulation 2). 


\section{EXPERIMENTS}

The trigonometric switch inputs law is experimentally tested with a prototype of the twowheeled mobile robot. We will compare the planning path of time polynomial inputs and trigonometric switch inputs.

Experiments are carried out according to simulations 1 and 2. In experiment 1, a straight line connecting the initial position and the desired position is taken as a reference line. In experiment 2, the reference line is connected by three non-collinear points of the initial position, the intermediate position and the desired position. The results of experiments 1 and 2 are shown in Figs. 10 and 11, respectively.

Experimental results show that the mobile robot can move to the desired positions. It is clear that the trigonometric switch inputs law is more efficient than polynomial inputs since the robot can accurately follow the reference using the former.

The trajectory of the mobile robot has a significant overshoot in experiment 1 with the polynomial inputs law because the planning path of the mobile robot is composed of the two basic movements of straight line motion and steering motion. On the contrary, although the movement of the robot, which is steered by trigonometric switch inputs law, is step-by-step, this motion method makes the planning path simple and efficient in practice. It also can be predicted that the trigonometric switch inputs law is more suitable for high coupling systems such as the $n$-trailer system and the manipulator system mentioned in $[3,8]$.
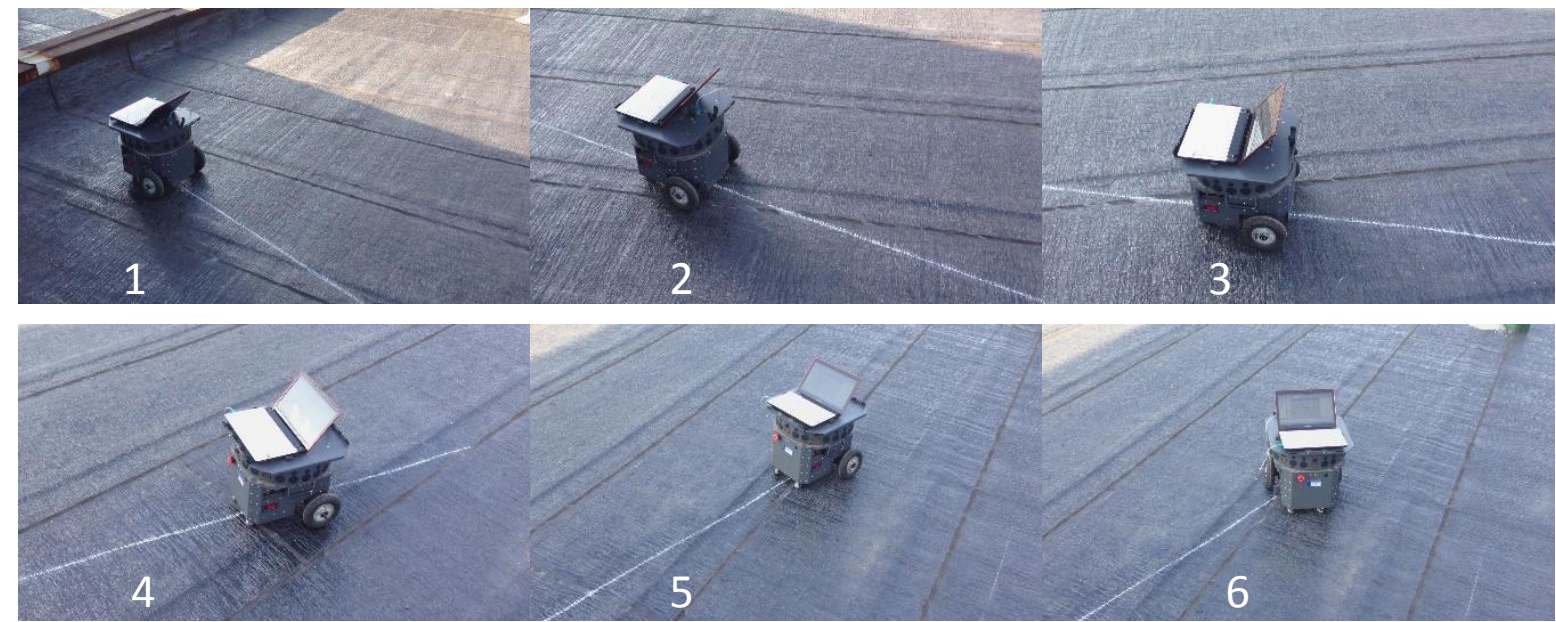

a) Experiment 1 with trigonometric switch inputs
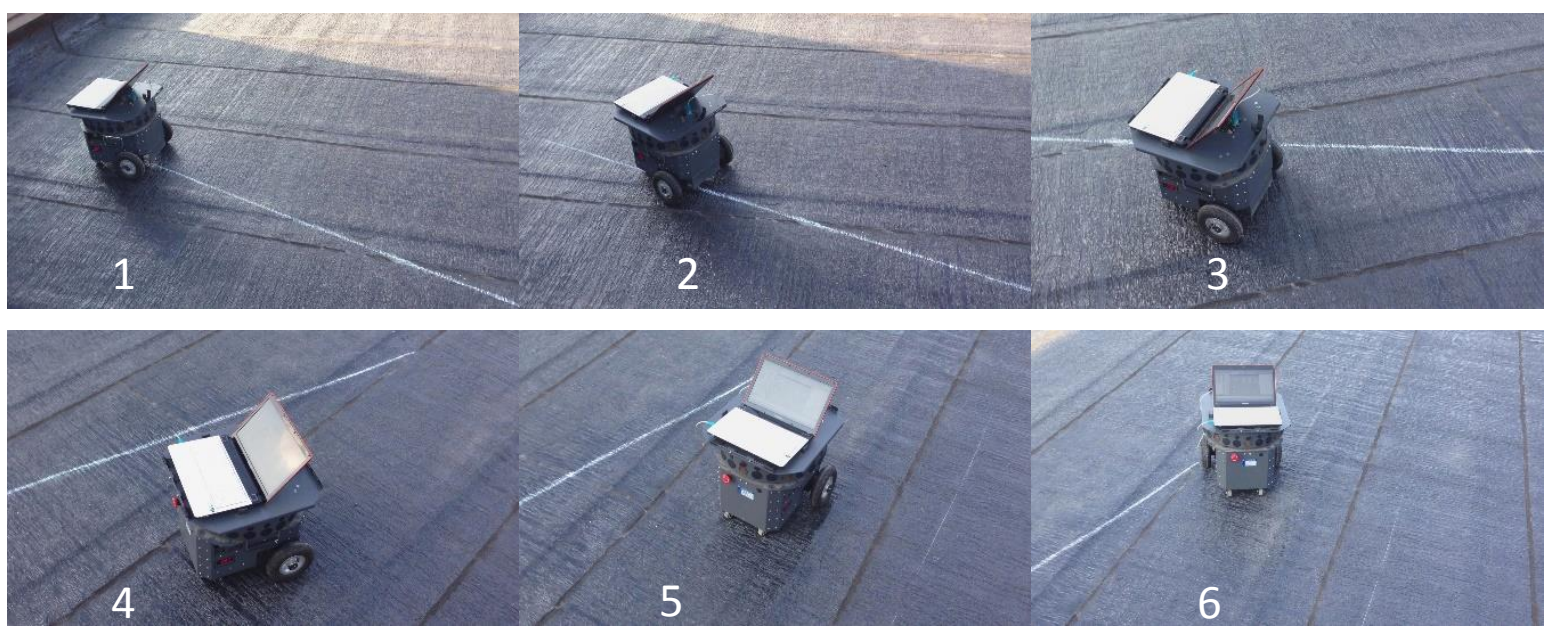

b) Experiment 1 with polynomial control

Figure 10: Experiment 1. 

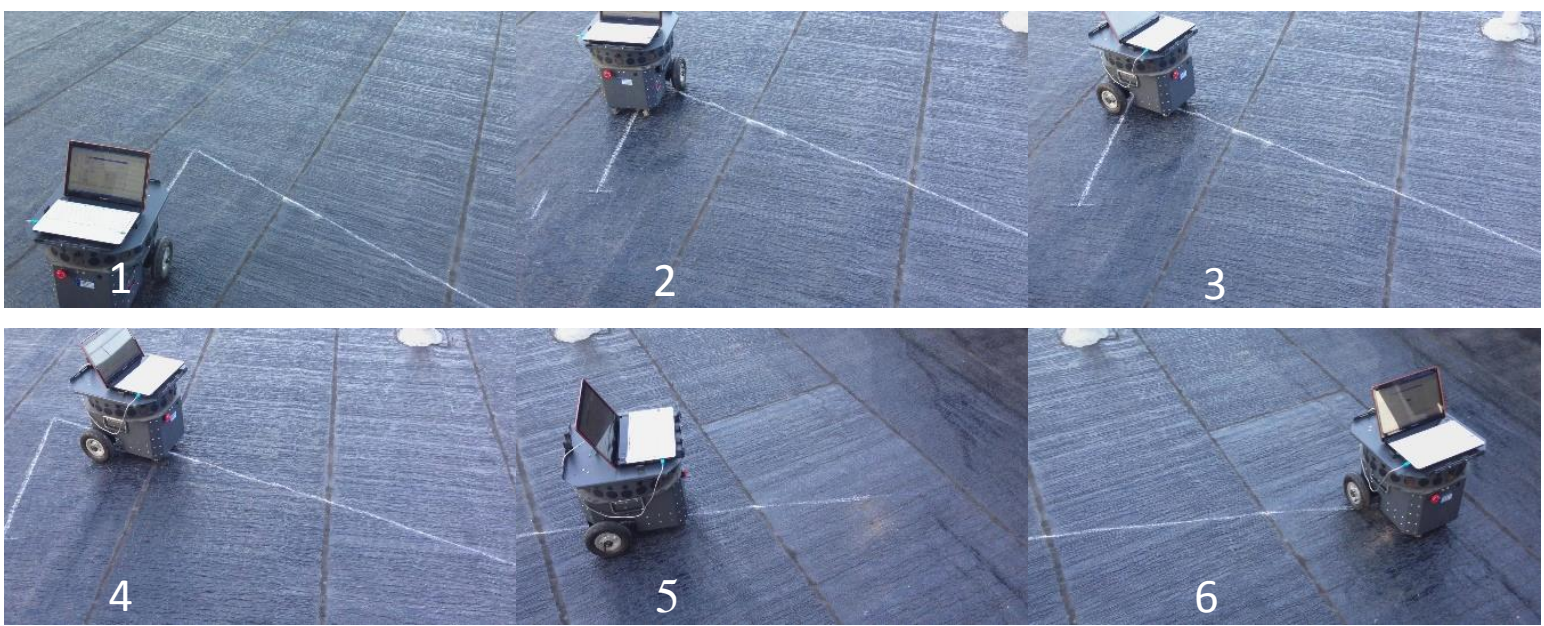

a) Experiment 2 with trigonometric switch inputs
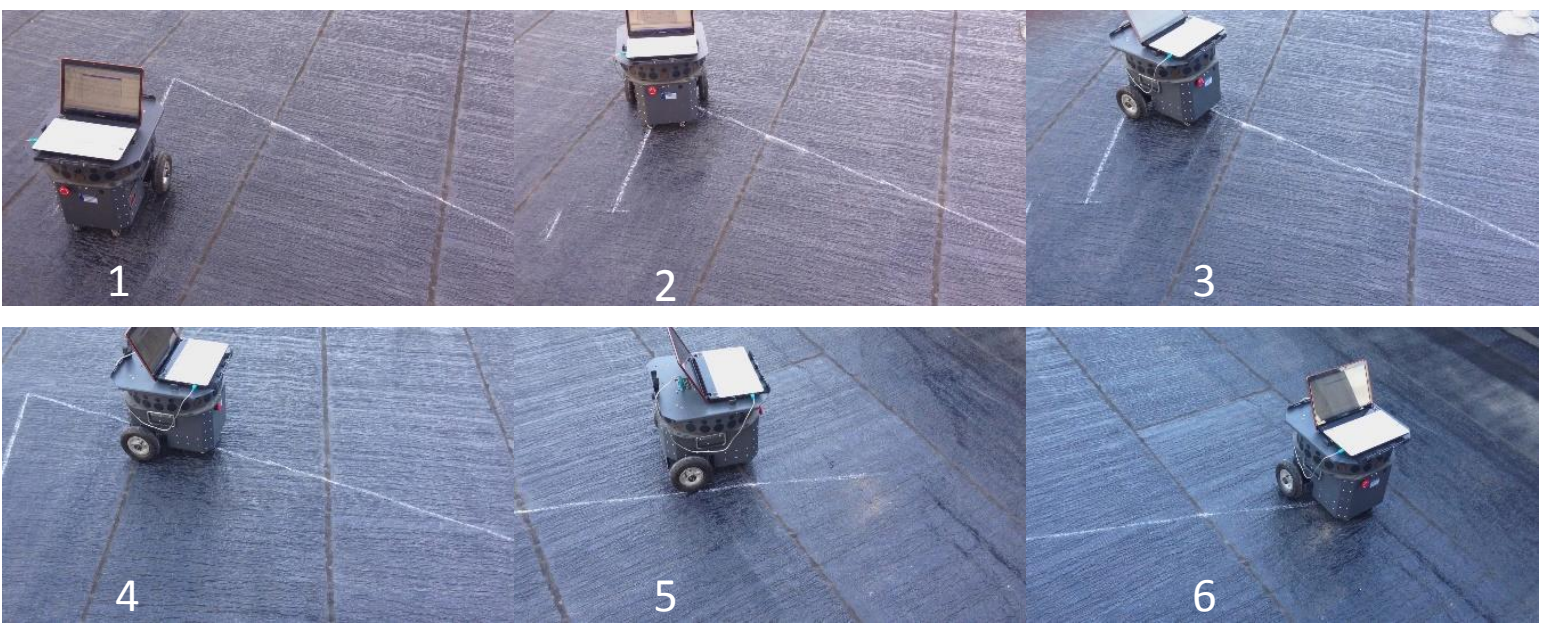

b) Experiment 2 with polynomial control

Figure 11: Experiment 2 (parallel parking).

\section{CONCLUSION}

In this paper, we present the trigonometric switch inputs law whose control inputs are stepby-step in order to simplify control. There are two major advantages of this motion planning law. One is that the planning path and curves of velocities inputs are smooth even in application of the time scale transformation technique. The other advantage is that trajectories have lower oscillation and computational costs. The two-wheeled mobile robot is taken as an example of trigonometric switch inputs. The simulation and experiment results show the validity of this motion planning law. Future research projects will include using this trigonometric switch inputs law to steer a nonholonomic manipulator with high dimensions and motion couplings.

\section{ACKNOWLEDGEMENT}

This work is supported by the Natural Science Foundation research project of Shaanxi Province (2016JM5019), the Science and Technology Plan Projects of Baoji City (16RKX1-30), and the Specialized Research Fund for the Doctor Program of Baoji University of Arts and Sciences (ZK16044). 


\section{REFERENCES}

[1] Suzuki, T.; Nakamura, Y. (1996). Planning spiral motion of nonholonomic space robots, Proceedings of the 1996 IEEE International Conference on Robotics and Automation, Vol. 1, 718-725, doi:10.1109/ROBOT.1996.503859

[2] Murray, R. M.; Sastry, S. S. (1991). Steering nonholonomic systems in chained form, Proceedings of the $30^{\text {th }}$ IEEE Conference on Decision and Control, Vol. 2, 1121-1126, doi:10.1109/CDC.1991.261508

[3] Sørdalen, O. J. (1993). Conversion of the kinematics of a car with $n$ trailers into a chained form, Proceedings of the 1993 IEEE International Conference on Robotics and Automation, Vol. 1, 382-387, doi:10.1109/ROBOT.1993.292011

[4] Tan, Y.-G.; Jiang, Z.-Q.; Zhou, Z.-D. (2006). A nonholonomic motion planning and control based on chained form transformation, Proceedings of the 2006 IEEE/RSJ International Conference on Intelligent Robots and Systems, 3149-3153, doi:10.1109/IROS.2006.282337

[5] Nakamura, Y.; Chung, W.; Sørdalen, O. J. (2001). Design and control of the nonholonomic manipulator, IEEE Transactions on Robotics and Automation, Vol. 17, No. 1, 48-59, doi:10.1109/70.917082

[6] Chen, C. C.; Li, J.-S.; Luo, J.; Xie, S.-R.; Li, H.-Y.; Pu, H.-Y.; Gu, J. (2016). Robust adaptive position and force tracking control strategy for door-opening behaviour, International Journal of Simulation Modelling, Vol. 15, No. 3, 423-435, doi:10.2507/IJSIMM15(3)3.342

[7] Murray, R. M.; Sastry, S. S. (1993). Nonholonomic motion planning: steering using sinusoids, IEEE Transactions on Automatic Control, Vol. 38, No. 5, 700-716, doi:10.1109/9.277235

[8] Tilbury, D.; Murray, R. M.; Sastry, S. S. (1995). Trajectory generation for the $n$-trailer problem using Goursat normal form, IEEE Transactions on Automatic Control, Vol. 40, No. 5, 802-819, doi: $10.1109 / 9.384215$

[9] Sekhavat, S.; Laumond, J.-P. (1998). Topological property for collision-free nonholonomic motion planning: the case of sinusoidal inputs for chained form systems, IEEE Transactions on Robotics and Automation, Vol. 14, No. 5, 671-680, doi:10.1109/70.720344

[10] Nakamura, Y.; Ezaki, H.; Tan, Y.-G.; Chung, W. (2001). Design of steering mechanism and control of nonholonomic trailer systems, IEEE Transactions on Robotics and Automation, Vol. 17, No. 3, 367-374, doi:10.1109/70.938393

[11] Li, L. (2016). Measurement and control system for underactuated manipulator based on LabVIEW, Revista de la Facultad de Ingeniería, Vol. 31, No. 3, 178-186, doi:10.21311/ 002.31 .3 .15

[12] Li, L.; Tan, Y.-G.; Li, Z. (2014). Nonholonomic motion planning strategy for underactuated manipulator, Journal of Robotics, Vol. 2014, Paper 743857, 10 pages, doi:10.1155/2014/743857

[13] Chelouah, A.; Di Giamberardino, P.; Monaco, S.; Norman-Cyrot, D. (1993). Digital control of nonholonomic systems: two case studies, Proceedings of the $32^{\text {nd }}$ IEEE Conference on Decision and Control, Vol. 3, 2664 -2669, doi:10.1109/CDC.1993.325680

[14] Li, S.; Ma, G.-L.; Chen, Q.-W.; Wu, X.-B.; Hu, W.-L. (2004). Nonholonomic motion planning: steering using bang-bang control, Proceedings of the 2004 American Control Conference, Vol. 5, 4653-4656

[15] Xu, W. L.; Ma, B. L.; Tso, S. K. (1999). Curve fitting approach to motion planning of nonholonomic chained systems, Proceedings of the 1999 IEEE International Conference on Robotics \& Automation, Vol. 1, 811- 816, doi:10.1109/ROBOT.1999.770074

[16] Deng, M.-C.; Inoue, A.; Sekiguchi, K. (2007). Parking control of a two wheeled mobile robot, Proceedings of the 2007 IEEE International Conference on Mechatronics and Automation (ICMA 2007), 539-544, doi:10.1109/ICMA.2007.4303600 